\title{
Optimising the release rate of naproxen liqui-pellet: a new technology for emerging novel oral dosage form
}

\author{
Matthew Lam ${ }^{1} \cdot$ Taravat Ghafourian $^{2} \cdot$ Ali Nokhodchi $^{1}$ (D)
}

Published online: 8 July 2019

(C) The Author(s) 2019

\begin{abstract}
Liqui-pellet is a new dosage form stemming from pelletisation technology and concept from liquisolid technology. In spite of liquipellet overcoming a major hurdle in liquisolid technology through achieving excellent flow property with high liquid load factor, the formulation requires to be optimised in order to improve drug release rate. Liqui-pellets of naproxen containing Tween 80, Primojel, Avicel and Aerosil were extruded and spheronised. Flowability test confirmed that all liqui-pellet formulations have excellent-good flow property (Carr's index between 3.9-11.17\%), including liqui-pellets with a high liquid load factor of 1.52, where 38\% of the total mass is co-solvent. This shows a relatively high liquid load factor can be achieved in liqui-pellet without compromising the flowability, which is one of the key novelty of this work. It was found that the improved drug release rate was due to the remarkably improved disintegration of the supposedly non-disintegrating microcrystalline-based pellet; the optimised liqui-pellet seems to explode into fragments in the dissolution medium. At $\mathrm{pH} 1.2$, the optimised formulation had $\sim 10 \%$ more drug release than non-optimised formulation after $2 \mathrm{~h}$, and at $\mathrm{pH} 7.4$, the drug release of the optimised pellet was nearing $100 \%$ at $\sim 15 \mathrm{~min}$, whereas the noneoptimised pellet only achieved $\sim 79 \%$ drug release after $2 \mathrm{~h}$. DSC and XRPD indicated an increase in the dissolution rate could be due to molecularly dispersion of naproxen in the pellets. Overall results showed that liqui-pellet exhibited an enhanced drug release and the capacity for high liquid load factor whilst maintaining excellent flowability, rendering it a potentially commercially feasible drug delivery system.
\end{abstract}

Keywords Liquisolid · Liquisolid pellet · Liqui-pellet · Dissolution enhancement · Extrusion-spheronisation · Liquid vehicle · Solid-state analysis

\section{Introduction}

Liqui-pellet is considered the novel emerging oral dosage form. It stems from liquisolid technology; however, liqui-pellet comes under liqui-mass system, which is different from liquisolid system. This liqui-mass system is combined with pelletisation technology, producing liqui-pellet [1]. In recent years, the advancement on the high-throughput screening has indicated an increased number of drug candidates to be either poorly water

Matthew Lam

matthew.lam.researcher@gmail.com

Ali Nokhodchi

a.nokhodchi@sussex.ac.uk

1 Pharmaceutics Research Laboratory, School of Life Sciences, University of Sussex, Brighton BN1 9QJ, UK

2 JMS Building, Biochemistry and Pharmacy Department, School of Life Sciences, University of Sussex, Brighton BN1 9QG, UK soluble or insoluble [2]. Since water-insoluble drugs have poor dissolution rate, the pharmaceutical industry has been challenged to overcome this in order to improve bioavailability [3]. In fact, approximately $40 \%$ of drugs in the market are considered poorly soluble in gastrointestinal fluids, and around $90 \%$ of drugs in development are identified as poorly soluble; both of which is based on biopharmaceutical classification system (BCS) [4].

Liqui-mass system (Fig. 1) is similar to liquisolid system in that it comprises a solubilised active pharmaceutical ingredient (API) in an appropriate co-solvent, which form the liquid medication. This liquid medication is absorbed and adsorbed into a carrier, which is usually microcrystalline cellulose. The admixture is then coated with a coating material that is usually nanosized silicon dioxide $[3,5]$. Other excipients, namely superdisintegrant, are usually added to improve dissolution rate. The major difference in liqui-mass system and liquisolid system is that the admixture of API and excipients is not necessarily a free-flowing powder in liqui-mass system, but instead can be a wet mass/paste. This is different from liquisolid system in which 


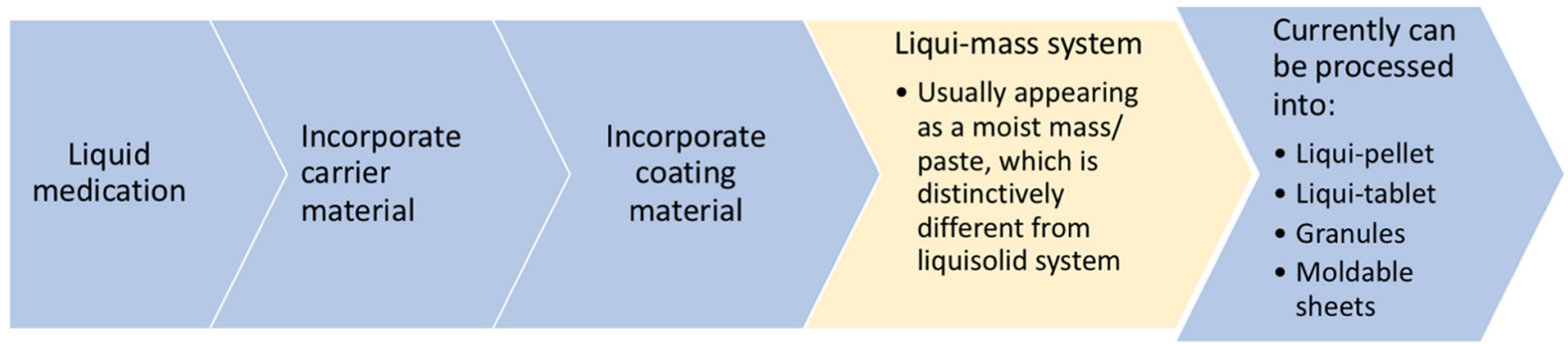

Fig. 1 Diagram summarising the novel liqui-mass system which is used to make liqui-pellet

it is made clear that the admixture as a free-flowing powder. It should be noted that liqui-pellet is different from liquisolid pellet in that liqui-pellet is under liqui-mass system. The name liquipellet also aid the distinction from liquisolid pellet in that liquipellet is capable of high liquid load factor.

It is noteworthy to point out that the technology involving liqui-mass system is highly versatile. It can be seen in Fig. 1 that liqui-mass system encompasses wet mass/paste and but less frequently free-flowing powder. In concept, the technology concerning liqui-mass system can produce free granule, moldable sheets, liqui-pellet, liqui-tablet and more, which will be revealed in future studies. There is also a considerable flexibility for modifications of the formulation, particularly the coating of liqui-pellet. Such versatility makes this new liqui-mass system interesting and exciting to explore. In addition, this technology has major advantages such as being costeffective, mainly uses green technology, simplistic approach, easy to scale up platform technology for commercial manufacturing, does not require organic volatile solvent, no need for an advance technique or machinery and excipient are common and easily obtainable [6]. Such advantages may not be present in other various technologies confronting the same issue of improving the bioavailability of poorly water-soluble drugs. Other technologies include conversion of crystalline drug into its amorphous state [7], solid dispersion [8]; micronisation [9-12], nanosuspension [13, 14], co-grinding [15-17], self-emulsifying drug delivery system $[18,19]$ and inclusion of drug solution in soft gelatin capsule [20]. It is claimed that the liqui-pellet is highly commercially feasible and has the potential to play a major role in the future oral dosage form [1].

The key purpose of liqui-pellet is to take the key advantages of liquisolid formulation into a commercially feasible dosage form. This is done by confronting the major drawbacks in liquisolid technology, such as poor flow property, poor compactibility and inability to produce high-dose drug without being too bulky and heavy for real-life use [3, 5]. In the authors' previous studies, it is shown that liqui-pellet can achieve excellent flow property whilst having a high liquid load factor of [1]. Liqui-pellet not only contains the advantages of liquisolid technology, but it also has the inherent advantages of being in a pellet form. Such advantages include good flow property [21], potential to combine incompatible drugs or drugs with different release profiles in same dose unit [22], reduced risk of side effects due to dose dumping and the flexibility for modification via coating technology.

The aim of the present study is to optimise liqui-pellet formulation in order to improve the drug release rate of naproxen, which is a nonsteroidal anti-inflammatory drug (NSAID), belonging to BSC class II. The chosen liquid vehicle used in the investigation is Tween 80 , as it was considered the most suitable liquid vehicle from previous studies [1]. Tween 80 solubilises the API as well as acting as a surfaceactive agent which reduces interfacial tension and improve water penetrating into the dosage form.

\section{Materials and methods}

\section{Materials}

Naproxen was obtained from Tokyo Chemical Industry Co (Japan). Other excipients used to prepare the liqui-pellet included microcrystalline cellulose (Avicel PH-101), (FMC corp., UK), hydrophilic fumed silica with specific surface area of $300 \mathrm{~m}^{2} / \mathrm{g}$ (Aerosil 300) (Evonik Industries AG, Hanau, Germany), sodium starch glycolate Type A (Primojel) (DFE Pharma, Goch, Germany), croscarmellose (Primellose) (DFE Pharma., Goch, Germany), 2-propanol (VWR Chemicals, Fontenay Sous Bois France), polysorbate 80 (Tween 80) and PEG with molecular weight of 1500 (Acros Organics, UK). All other reagents and solvent were of analytical grades.

\section{Preparation of naproxen liqui-pellet}

The pestle and mortar method was applied to mix naproxen in Tween 80 to form the liquid medication. Avicel PH-101 (carrier) and Aerosil 300 (coating materials) were used in all the formulation with a ratio of carrier to coating material of 20 . The liquid medication was then incorporated into Avicel before transferring into a mixer (Caleva Multitab, Caleva Process Solutions Ltd., UK), where the sample was mixed for $10 \mathrm{~min}$ at a constant rate of $125 \mathrm{rpm}$ with deionised water added bit by bit to achieve reasonable plastic property for 
extrusion (Caleva Multitab, Caleva Process Solutions Ltd., UK). Aerosil 300 was then added into the admixture and further mixed for $10 \mathrm{~min}$ before extrusion. Once the sample was extruded, the extrudate was spheronised at an almost constant rotation at $4000 \mathrm{rpm}$ (decrease to $3500 \mathrm{rpm}$ if agglomeration seemed likely or increase to $4500 \mathrm{rpm}$ to increase pellet sphericity). The spheronisation time varied depending on the extrudate plastic property to avoid agglomeration. Pellets were then placed in an oven under a constant temperature of $50{ }^{\circ} \mathrm{C}$ overnight to remove water content.

For clarity, it should be mentioned that the liqui-pellet formulations were categorised into two sections (Table 1). In the first section between LP-1 to LP-6, the main focus was to look into the effect of varying concentration of a Primojel (superdisintegrant) with and without the presence of Tween 80. In the second section of the formulations between LP-7 and LP-11, the focus was to modify the formulations in an attempt to improve drug release rate.

\section{Evaluation of naproxen liqui-pellet}

\section{Flowability test on liqui-pellet}

Three techniques were used to evaluate the flow properties of the liqui-pellet formulations, namely flow rate in grams per second (Flowability tester, Copley Scientific, UK), angle of repose (Flowability tester, Copley Scientific, UK and Digimatic height gauge, Mitutoyo, Japan) and Carr's compressibility index using the SVM tapped density tester (D-63150, Erweka, Germany). Flow rates were measured by recording the weight $(\mathrm{g})$ and time (s) of pellets flowing through a 10 -mm-diameter orifice after applying the shutter. Note that shutter was applied before the funnel holding the pellet became empty. To determine the angle of repose, the pellets were placed in a funnel with 10 -mm-diameter orifice and let the pellets flow onto a 100 -mm-diameter circular test platform. The digimatic height gauge and micrometre were used to measure the height and diameter of the heap of the sample, so that the angle of repose could be determined. Carr's compressibility index (CI\%) was calculated using $\mathrm{CI}$ equation. Tapped density was measured using the tapped density tester, which was set to tap 100 times. All measurements were done in triplicates.

\section{Friability test on of liqui-pellet}

The friability test was adapted using a similar method as in $\mathrm{Hu}$ studies [23]. Two best-optimised formulations were tested. Pellets $(3 \mathrm{~g})$ and glass beads $(3 \mathrm{~g})$ were placed in Erweka friabilator (D-63150, Erweka, Germany) and sealed to prevent pellets from leaving the container. The friabilator was then rotated under the constant speed of $25 \mathrm{rpm}$ for $4 \mathrm{~min}$.
Weight of the pellets before and after the friability test was recorded and used to calculate the percentage of weight loss.

\section{Particle size analysis via sieve method}

The particle size distribution was determined using sieve method (Test sieve, Retsch, Germany). Only the physical mixture pellet and two chosen optimised formulations were tested. Pellets ( $5 \mathrm{~g}$ ) were sieved under vibration using the mechanical shaker (AS 200, Retsch, Germany) for 1 min with an amplitude of 50, then a further 9 min with an amplitude of 40 . The size of sieves used were 2000-, 1000-, 850-, 500- and 250- $\mu \mathrm{m}$ sieves. The pellets yield was determined based on the pellet fraction between 250 and $2000 \mu \mathrm{m}$ and presented as the percentage of total pellet weight.

\section{Stereoscopic analysis}

Mean Feret's diameter along with mean roundness and mean elongation ratio was determined using an optical microscope (Nikon Labophot, Nikon, Japan) connected to a camera (Panasonic camera WVCL310, Panasonic, Japan). The image of a pellet was captured and processed using particle size analysis software V1999 (designed in-house at King's College London). This was repeated so that 100 pellets were analysed per formulation in order to obtain Feret's diameter and also shape factors such as roundness and elongation ratio. Roundness and elongation ratio were calculated using Eqs. 1 and 2 respectively [24]. Only physical mixture pellet and two best-optimised formulations underwent stereoscopic analysis.

Roundness $=(\text { perimeter })^{2} /(4 \pi A)$

Elongation ratio $=$ Maximum Feret diameter $/$ Minimum Feret diameter

\section{Scanning electron microscope analysis}

Surface morphology studies of the liqui-pellet from each formulation were carried out using a scanning electron microscope (Jeol JMS 820, Freising, Germany). The samples were placed on a double-sided carbon tape and sputter-coated with gold using a sputter coater (Edwards S-150 sputter coater, Edwards High Vacuum Co. International, USA) with gold target and Argon gas under $5 \mathrm{kV}$ for $5 \mathrm{~min}$. After the samples were coated, it was ready to be placed into the scanning electron microscope (SEM) where surface structure was then observed and recorded at magnifications of $\times 80, \times 200$ and $\times$ 800 , using the SEM which was operating at $3 \mathrm{kV}$. 


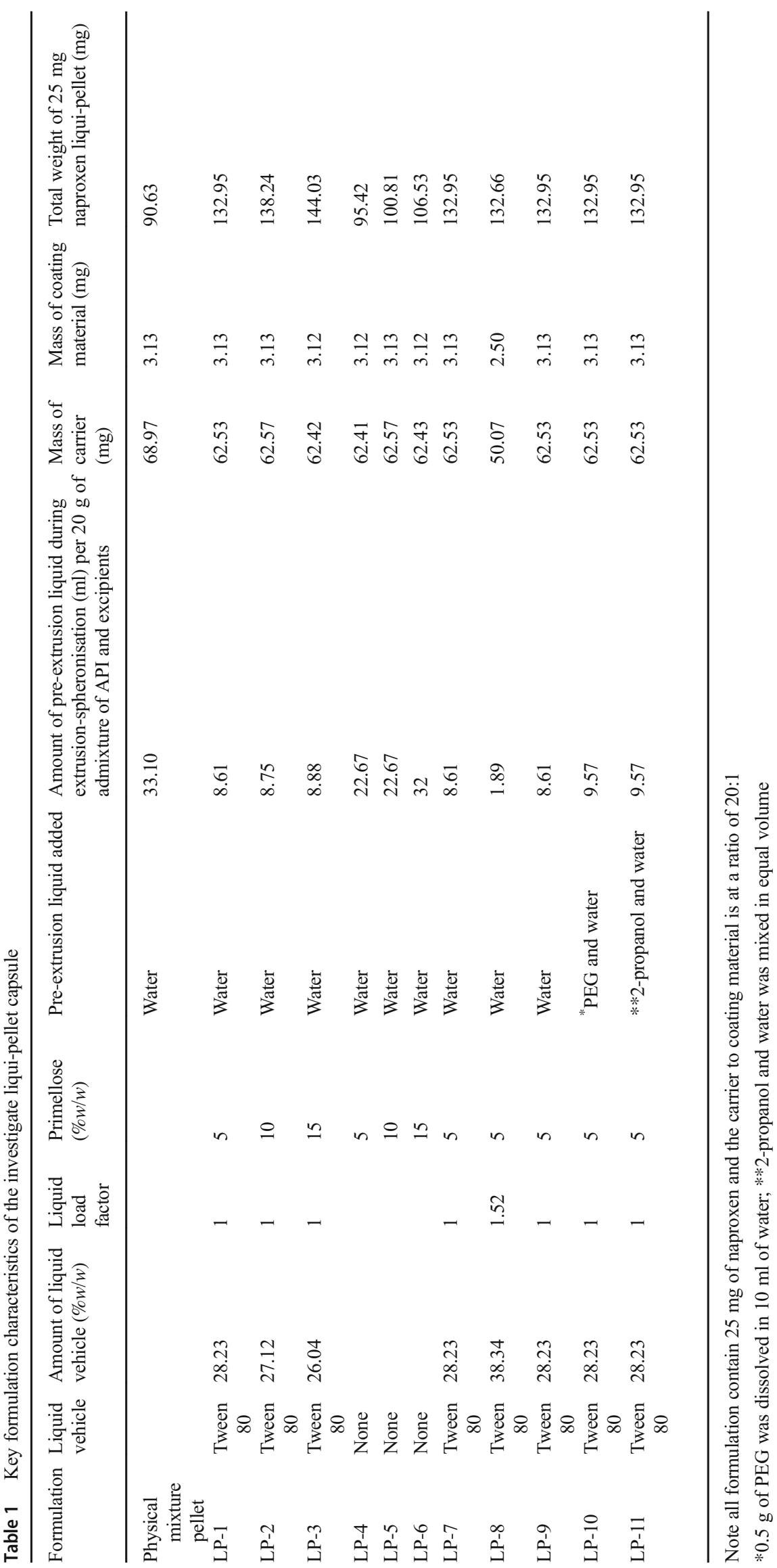




\section{In vitro drug dissolution test}

The USP dissolution paddle method (708-DS Dissolution Apparatus \& Cary 60 UV-Vis, Agilent Technologies, USA) was carried out for all formulations. All dissolution tests of the pellets in capsule were under the constant condition of $900 \mathrm{ml}$ dissolution medium, paddle rotating at $50 \mathrm{rpm}$ and temperature of $37.3 \pm 0.5^{\circ} \mathrm{C}$.

Dissolution media that were used were either $\mathrm{HCl}$ buffer solution of $\mathrm{pH} 1.2$ or phosphate buffer solution of $\mathrm{pH} 7.4$ to simulate gastric fluid or intestinal fluid respectively without enzymes. The absorbance of the samples was taken at time intervals of $5 \mathrm{~min}$ for $1 \mathrm{~h}$, and then time interval of $10 \mathrm{~min}$ for another hour. The authors were aware that at $\mathrm{pH} 1.2$, the sink condition was not maintained; however, this $\mathrm{pH}$ was only used for comparison of various formulations.

All formulations contained $25 \mathrm{mg}$ of naproxen and this amount of naproxen was chosen due to its poor solubility profile at $\mathrm{pH}$ 1.2. This weakly acidic drug would need to be able to dissolve completely at $\mathrm{pH} 1.2$ in order for the dissolution test to be considered reliable. This value was also taken on the basis of its solubility at $35{ }^{\circ} \mathrm{C}$ and $\mathrm{pH} 1.2$ which was $27 \mathrm{mg} / \mathrm{l}$ [25]. Although it does not follow the sink conditions, it can be used for comparison purpose for dissolution profiles of different formulations. Thus, $25 \mathrm{mg}$ of naproxen seemed reasonable in this test. At $\mathrm{pH} 7.4$, naproxen was extremely soluble with a solubility of $\sim 3347 \mathrm{mg} / \mathrm{l}$; hence, using $25 \mathrm{mg}$ of the drug was not a problem.

\section{Solid-state analysis}

\section{Differential scanning calorimetry studies}

Differential scanning calorimetry (DSC) (DCS 4000, Perkin Elmer, USA) was performed on the chosen liqui-pellet formulations with the fastest dissolution rate, including its excipients and pure naproxen in order to indirectly assess the solid state of naproxen in the formulations using data from thermograms. Samples weighing between 3 and $6 \mathrm{mg}$ were sealed in aluminium pan and placed in the DSC machine with a scanning rate of $10{ }^{\circ} \mathrm{C} / \mathrm{min}$ (from 25 to $200{ }^{\circ} \mathrm{C}$ ) under nitrogen atmosphere.

\section{X-ray powder diffraction studies}

X-ray powder diffraction (XRPD) was performed using X-ray diffractometer (D5000, Siemens, Germany) on naproxen, excipients and selected optimised formulations in order to characterise the solid state of the materials used. Samples were scanned over a range of $2 \theta$ at voltage of $40 \mathrm{kV}$ and current of $30 \mathrm{~mA}$, with scanning angle ranged from $5^{\circ}$ to $40^{\circ}$ and scan rate of $0.2 \%$.
The percent relative crystallinity was calculated using integrated peak method (Eq. 3) and peak height method (Eq. 4) [26]. Trapezoid method was applied to calculate the area under the curve for integrated peak method.

$\% \mathrm{XRD}$ relative crystallinity $=\left(A_{\mathrm{S}} / A_{\mathrm{r}}\right) \times 100$

$\% \mathrm{XRD}$ relative crystallinity $=\left(H_{\mathrm{s}} / H_{\mathrm{r}}\right) \times 100$

\section{Statistical and mathematical analysis}

Mean cumulative $\%$ drug release after $2 \mathrm{~h}$ from the dissolution test was statistically analysed by one-way analysis of variance (ANOVA). Results were quoted as significant where $p<0.05$.

Difference factor $\left(f_{1}\right)$, Eq. 5 , and similarity factor $\left(f_{2}\right)$, Eq. 6 [27] were used to determine the differences/similarities in terms of dissolution profile among the formulations. Such methods have been recommended by the US FDA (Food and drug administration) [28] and implemented by the FDA in various guidance documents $[29,30]$. When $f_{1}$ value is between 0 and 15 and $f_{2}$ value is between 50 and 100 , this indicates equivalence of the two dissolution profiles [31]. Details of the equations can be found in various literature [28, 32-34]. In brief, $n$ represents the number of dissolution sample times and $R_{t}$ and $T_{t}$ represent the mean $\%$ of drug dissolved at each time point $(t)$.

$$
\begin{aligned}
& f_{1}=\left\{\left[\sum_{t=1^{n}}\left|R_{t}-T_{t}\right|\right] /\left[\sum_{t=1^{n}} R_{t}\right]\right\} \cdot 100 \\
& f_{2}=50 \cdot \log \left\{\left[1+(1 / n) \sum_{t=1^{n}}\left(R_{t}-T_{t}\right)^{2}\right]^{-0.5} \cdot 100\right\}
\end{aligned}
$$

\section{Results and discussion}

\section{Evaluation of naproxen liqui-pellet}

\section{Liqui-pellet flow property}

Results from the flowability tests are shown in Table 2. It is clear that all formulations have excellent or in the borderline between excellent to good flow property. Thus, it is claimed that liqui-pellet is a promising dosage form, which resolves poor flowability issue in liquisolid technology and yet maintains the inherent advantages stemming from liquisolid concept. Pezzini et al. have applied liquisolid system to pellet, but in the current study, liqui-mass system was used instead [35]. Both liqui-pellet and liquisolid pellet do indeed contain inherent advantage from liquisolid and pelletisation technologies. However, they are distinctively different in that they both use different systems (i.e. liqui-mass system versus liquisolid system). The potential of Liqui-pellet is that liquid load factor is 
Table 2 Flow rate (g/s), angle of repose and Carr's compressible index (CI\%) of all liqui-pellet formulation $(n=3)$

\begin{tabular}{llllll}
\hline Formulation & $\begin{array}{l}\text { Flow rate } \\
(\mathrm{g} / \mathrm{s}) \pm \mathrm{SD}^{\mathrm{b}}\end{array}$ & Angle of repose $\pm \mathrm{SD}^{\mathrm{b}}$ & $\mathrm{CI} \% \pm \mathrm{SD}^{\mathrm{b}}$ & Inference according to angle of repose & Inference according to CI\% \\
\hline Physical mixture pellet & $8.02 \pm 0.24$ & $27.95 \pm 0.14$ & $9.08 \pm 0.87$ & Excellent flow property & Excellent flow property \\
LP-1 & $7.60 \pm 0.10$ & $26.98 \pm 0.74$ & $5.25 \pm 0.86$ & Excellent flow property & Excellent flow property \\
LP-2 & $7.61 \pm 0.12$ & $27.75 \pm 0.31$ & $8.13 \pm 1.65$ & Excellent flow property & Excellent flow property \\
LP-3 & $7.42 \pm 0.22$ & $28.68 \pm 0.53$ & $6.07 \pm 1.44$ & Excellent flow property & Excellent flow property \\
LP-4 & $10.68 \pm 0.06$ & $23.81 \pm 0.40$ & $9.95 \pm 0.08$ & Excellent flow property & Excellent flow property \\
LP-5 & $8.59 \pm 0.08$ & $28.20 \pm 0.16$ & $11.17 \pm 0.85$ & Excellent flow property & Good flow property \\
LP-6 & $6.96 \pm 0.28$ & $29.21 \pm 0.26$ & $10.37 \pm 0.79$ & Excellent flow property & Excellent-good flow property \\
LP-7 & $7.13 \pm 0.07$ & $28.68 \pm 0.22$ & $7.24 \pm 2.33$ & Excellent flow property & Excellent flow property \\
LP-8 & $5.82 \pm 0.09$ & $30.51 \pm 0.38$ & $3.90 \pm 2.30$ & Excellent-good flow property & Excellent flow property \\
LP-9 & $7.35 \pm 0.05$ & $28.57 \pm 0.50$ & $7.63 \pm 1.42$ & Excellent flow property & Excellent flow property \\
LP-10 & $6.47 \pm 0.19$ & $30.13 \pm 0.19$ & $9.24 \pm 0.73$ & Excellent-good flow property & Excellent flow property \\
LP-11 & $6.03 \pm 0.25$ & $30.47 \pm 0.51$ & $7.76 \pm 0.76$ & Excellent-good flow property & Excellent flow property \\
\hline
\end{tabular}

${ }^{\mathrm{a}}$ For the composition of each formula refer to Table 1

${ }^{\mathrm{b}} S D$, standard deviation from the mean

considerably higher than what liquisolid pellet can ever achieve.

The previous studies by the authors demonstrated that liqui-pellets achieved a high liquid load factor of 1 , whilst maintaining excellent flow property [1]. In fact, before the development of liqui-pellet, it has been proven very difficult to achieve such mentioned results, due to the cohesive nature of liquid powder admixture, which is shown in various studies [36-38]. Tiong et al. formulated naproxen liquisolid powder with $L_{\mathrm{f}}$ of 0.9 but the flowability was poor (Carr's index of 31.58) [36]. In studies by Javadzadeh et al., an additive such as PEG 3500 was used to increase the $L_{\mathrm{f}}$ [37]. They observed an increase of carbamazepine $L_{\mathrm{f}}$ from 0.25 to 0.6 [37]; however, it is clear that liqui-pellet $L_{\mathrm{f}}$ is much more superior and does not need polymeric additive. Hentzschel et al. replaced a commonly used carrier (Avicel) and coating material (Aerosil) with neusilin, a material with a much larger specific surface area (SSA) [38]. This enabled an increase of $L_{\mathrm{f}}$ by a factor of $\sim 7$; nonetheless, it is still limited by its flow property; their formulations' flow rate are below $1 \mathrm{~g} / \mathrm{s}$ [38].

What is exciting and promising in liqui-pellet is that it can be further optimised so that the $L_{\mathrm{f}}$ is further increased. It can be seen in Table 1 that LP- 8 has $L_{\mathrm{f}}$ as high as 1.52 , where $38 \%$ of the pellet total mass is co-solvent, and yet excellent-good flow property is achieved. Such a result further supports the

Table 3 Weight loss of $3 \mathrm{~g}$ of each formulation under rotational speed of $25 \mathrm{rpm}$ for $4 \mathrm{~min}$

\begin{tabular}{ll}
\hline Formulation & \% weight loss \\
\hline Physical mixture pellet & 0.54 \\
LP-8 & 0.03 \\
LP-11 & 0.12 \\
\hline
\end{tabular}

potential of liqui-pellet being commercially feasible. This means flow property and $L_{\mathrm{f}}$ are not a major hindrance for liqui-pellets; this emerging novel oral dosage form has the potential for smooth and cost-effective manufacturing, as well as producing high-dose liqui-pellet without being overly bulky and heavy if the formulation is optimised. Although Pezzini et al. [35] overcome the flow issue of liquisolid by converting it to pellet form, the load factor calculated was around 0.1 (they did not calculate the load factor and the authors of the current research calculated it on the basis of mass data provided in Pezzini's article), which is much lower than the load factor obtained in the current article $\left(L_{\mathrm{f}} \geq 1\right)$. This indicates that at the same concentration of API, the final formulation in Pezzini's article should be very bulky. In addition to load factor, the percentage of API in Pezzini formulation was around 5\% (percentage of API was calculated on the basis of mass data provided in Pezzini's article). This is lower than the majority of liqui-pellet formulation, which is around 19\% (percentage of API was calculated on the basis of mass data in Table 1).

\section{Friability test}

Table 3 shows the results obtained from the friability test of the physical mixture pellet and two optimised formulations (LP-8 and LP-11). In brief, they all have percentage weight loss below $1 \%$. Since there is currently no standard for friability test on pellets, USP standard friability test for tablet is adapted, which suggests less than $1 \%$ weight loss is acceptable. Therefore, it can be concluded that all tested formulations are robust, which is ideal for commercial manufacturing in terms of quality control. 

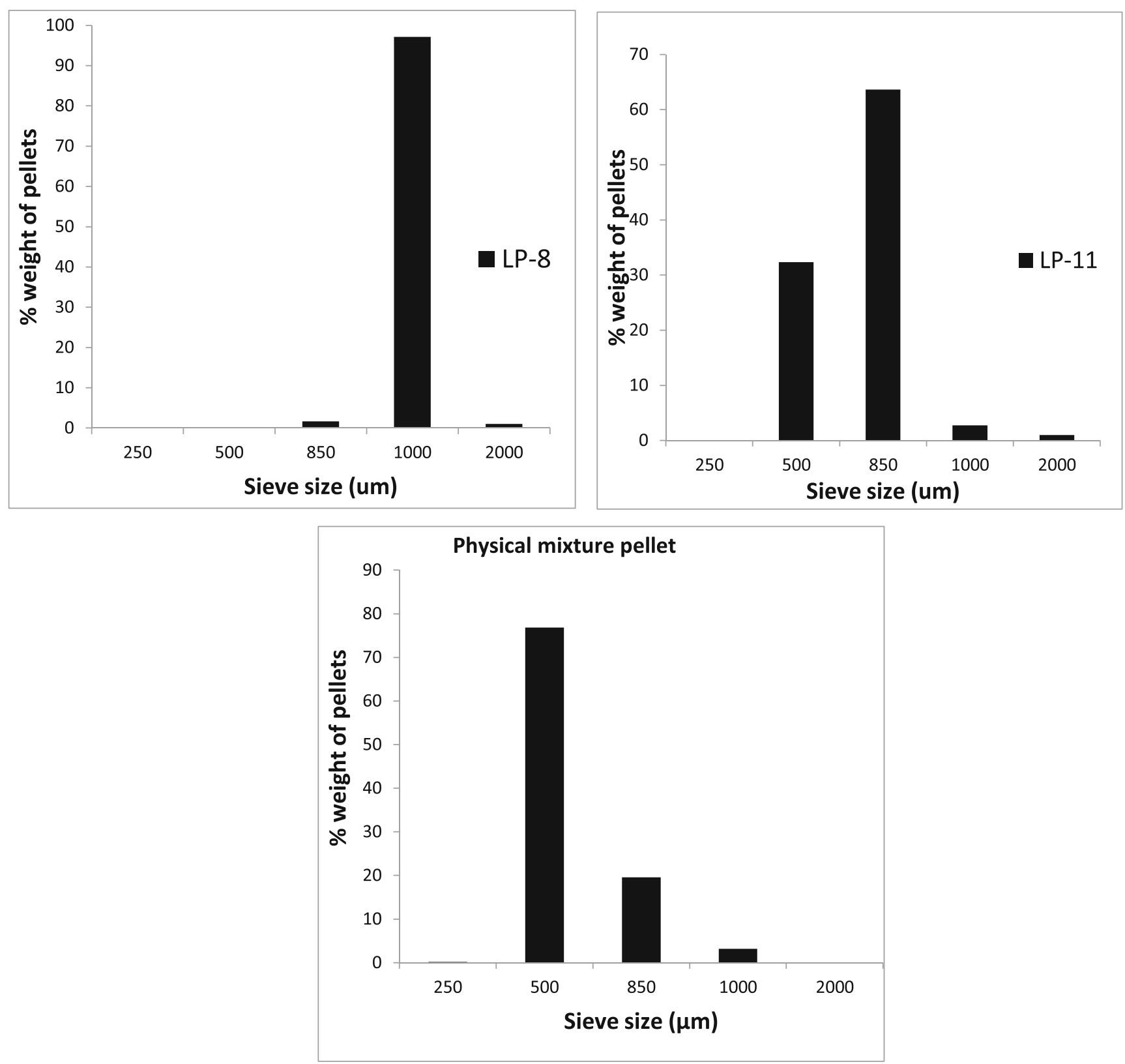

Fig. 2 Particle size distribution of LP-8 (top), LP-11 (middle) and physical mixture pellet (bottom)

It can also be postulated that the robustness is due to microcrystalline cellulose (carrier) forming sufficient bonds within its structure when water is added, hence producing robust pellets.
In addition, the Tween 80 in the liqui-pellet can increase the pellet plasticity due to plasticising effect [39], which effectively increases the pellet resistant to friability.
Table 4 Stereoscopic analysis showing the mean Feret's diameter, mean roundness and mean elongation of physical mixture pellet and optimised formulation $(n=100)$

\begin{tabular}{llll}
\hline Formulations $^{\mathrm{a}}$ & $\begin{array}{l}\text { Mean Feret's diameter } \\
(\mathrm{mm})\end{array}$ & $\begin{array}{l}\text { Mean roundness } \pm \\
\mathrm{SD}^{\mathrm{b}}\end{array}$ & $\begin{array}{l}\text { Mean elongation ratio } \pm \\
\mathrm{SD}^{\mathrm{b}}\end{array}$ \\
\hline Physical mixture pellet & 1.028 & $1.25 \pm 0.12$ & $1.41 \pm 0.19$ \\
LP-8 & 1.431 & $1.28 \pm 0.14$ & $1.43 \pm 0.21$ \\
LP-11 & 1.527 & $1.42 \pm 0.12$ & $1.95 \pm 0.35$ \\
\hline
\end{tabular}

${ }^{\text {a }}$ For the composition of each formula, refer to Table 1

${ }^{\mathrm{b}} S D$, standard deviation from the mean 


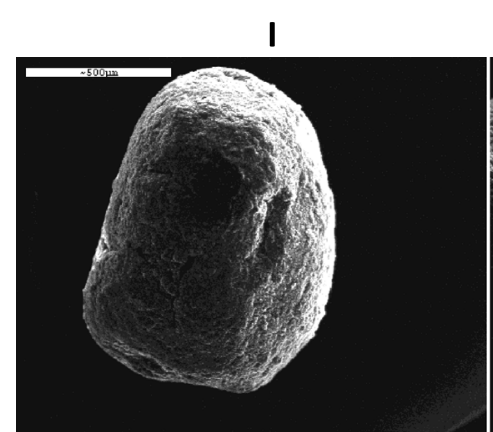

II

III
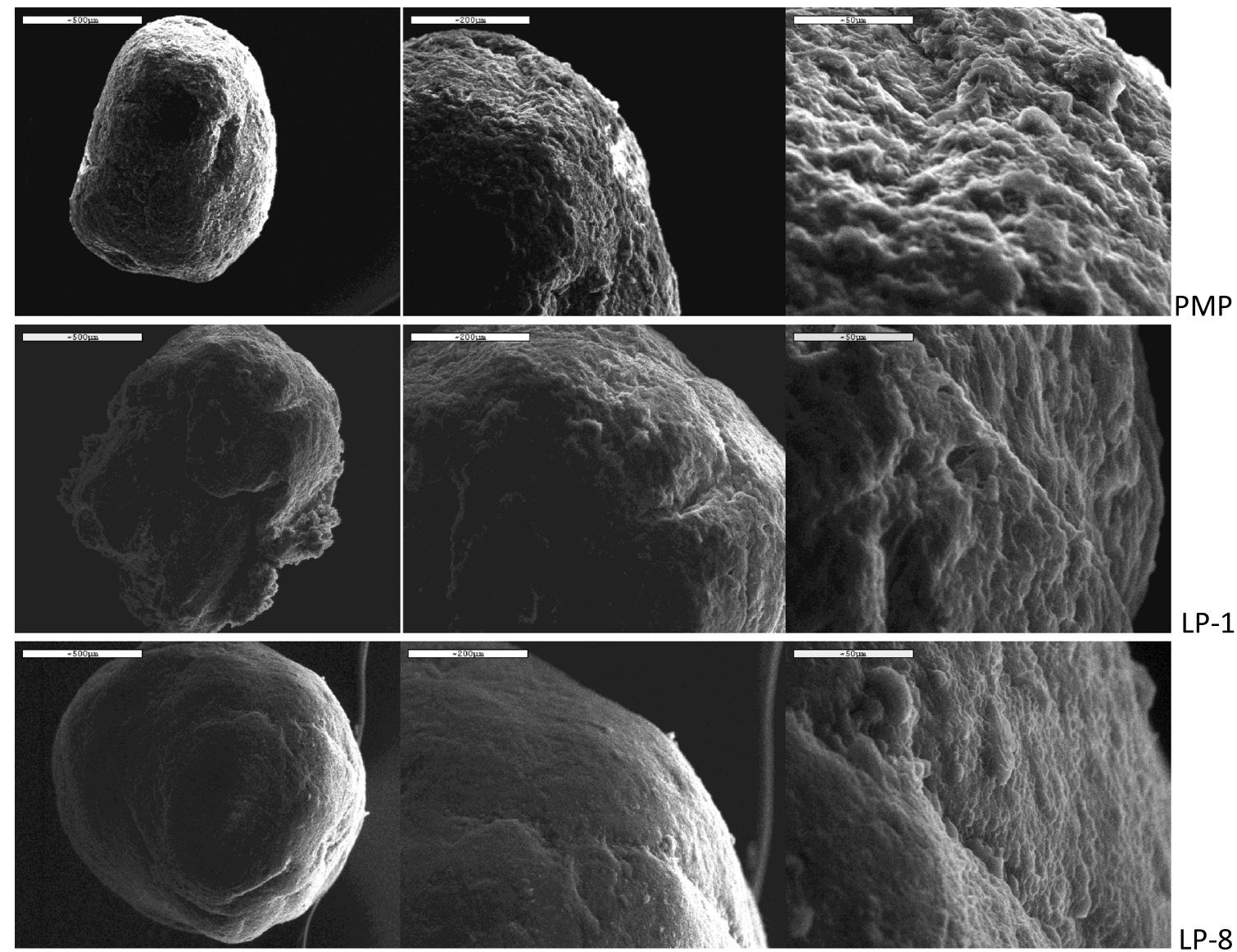

LP-1
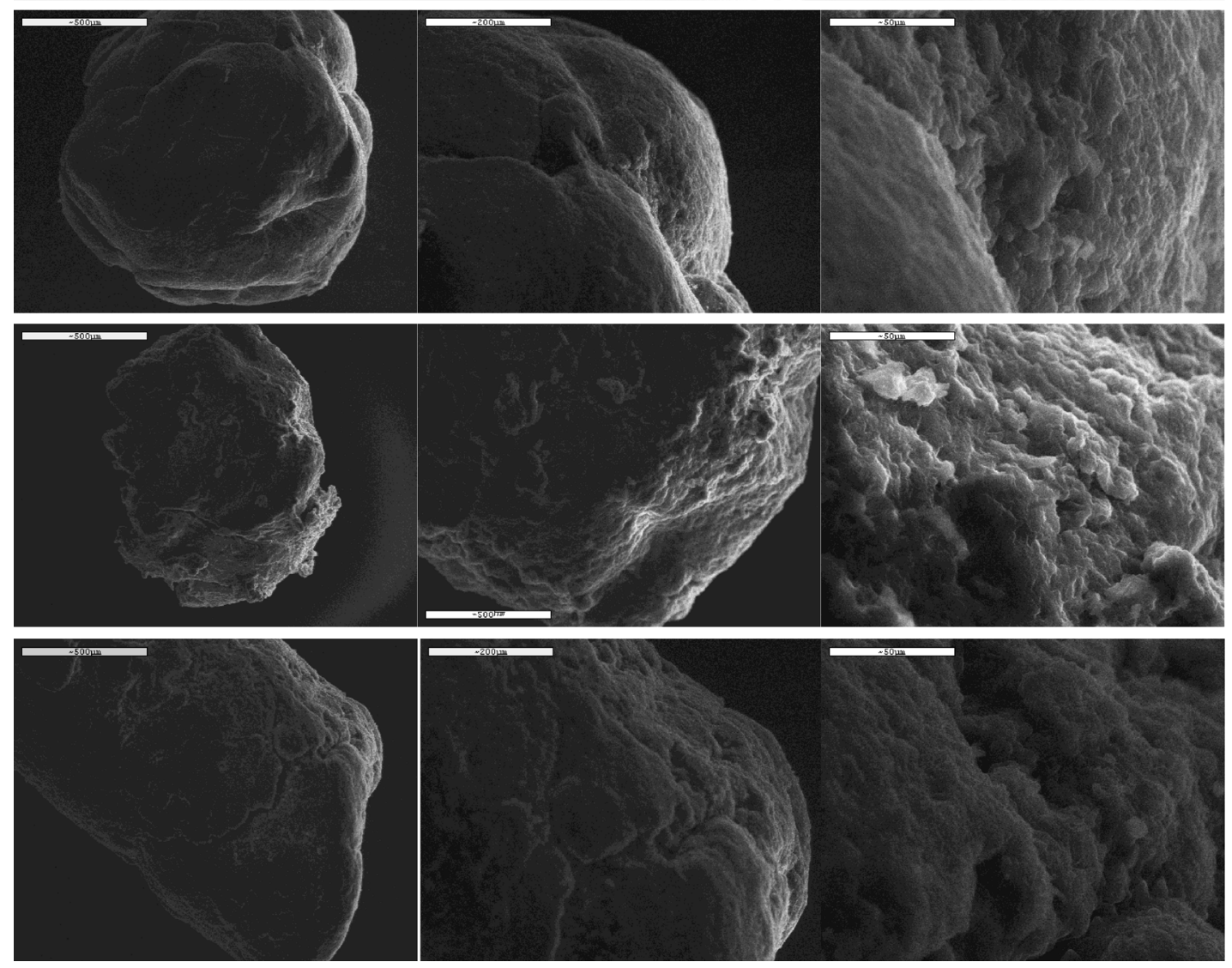
Fig. 3 Images from SEM of physical mixture pellet, LP-7, LP-8 and LP11 ; (I) $\times 80$ magnification, (II) $\times 200$ magnification and (III) $\times 800$ magnification

\section{Particle size analysis via sieve method}

Results from particle size analysis of the optimised formulation LP-8 (Fig. 2) indicate that $\sim 97 \%$ of the pellets fall in the size of $1 \mathrm{~mm}$. This shows that the optimised liqui-pellet LP-8, have a good uniformity of size. This is ideal in perspective of manufacturing. There will be less likelihood of variation in volume of pellet during encapsulation, consequently maintaining uniformity of drug content during filling process.

Particle size analysis of LP-11 shows a wider size distribution with $\sim 64 \%$ fall in the size of $850 \mu \mathrm{m}$ and $\sim 32 \%$ fall in the size of $1 \mathrm{~mm}$. This could be due to the reduced plastic property as a result of a reduction in water content, leading to a poorer quality pellet with wider size distribution.

Nonetheless, both of the optimised formulations are almost entirely below 2-mm range which will behave similarly to liquid in the stomach and be emptied into the small intestine relatively fast [40]. This can be beneficial for weakly acidic drugs (i.e. naproxen), as they are more soluble in an alkaline environment, suggesting that bioavailability and onset of action may be improved.

It is also interesting to note that most of the physical mixture pellet falls in $500 \mu \mathrm{m}$, which is considered small. This supports the claim from the previous studies by the authors that co-solvent tends to increased pellet size [41].

\section{Stereoscopic analysis}

The Feret's diameter (Table 4) agrees with the trend that cosolvent tends to increase liqui-pellet size. It can be seen that physical mixture and LP-11 mean Ferret diameter overestimated the liqui-pellet size. Since the pellets are not perfectly spherical, they tend to be in their most stable orientation. This means that the smallest dimension is orientated vertically; therefore, overestimation is prone to occur [42].

It is clear that LP-11 is the least round with mean roundness deviating from 1 considerably (1.42). Its mean elongation ratio is also large which supports the visible observation of the cylindrical liqui-pellet. Perhaps the reduced plastic property due to decreased water content leads to incomplete spheres forming. Nonetheless, LP-11 has excellent-good flowability.

\section{Scanning electron microscope analysis}

The SEM results (Fig. 3) show physical mixture pellet (PMP) has the roughest surface structure compared to the rest of the formulations. This can be seen clearly at $\times 800$ magnification. Although formulation LP-1, which contains $28 \%$ Tween 80 , shows surface crack at $\times 80$ magnification, its surface is less rough than the physical mixture pellet. This is more apparent

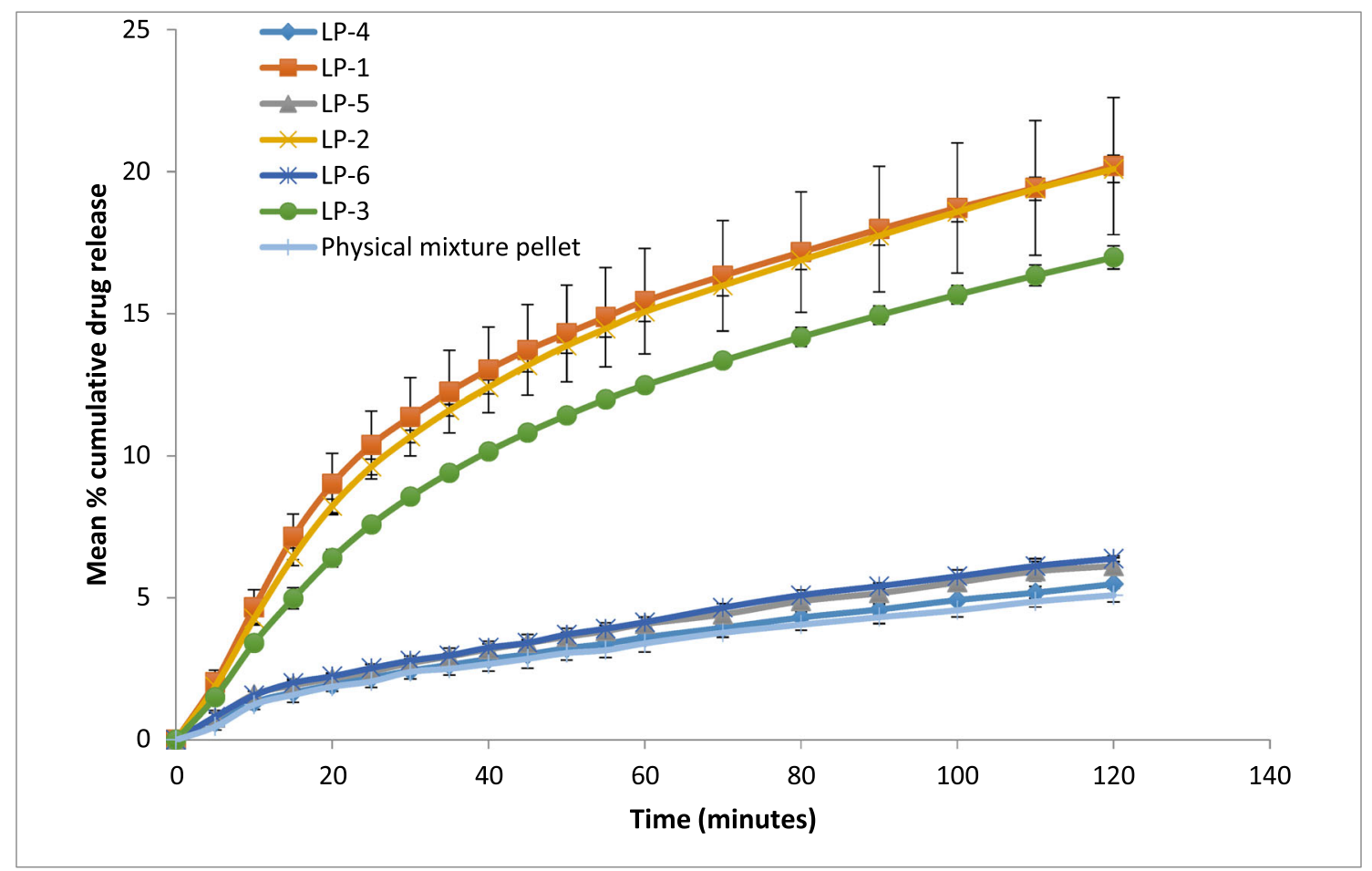

Fig. 4 Dissolution profile of pellets in capsule for naproxen $25 \mathrm{mg}$ with different concentrations of Primojel $(5,10$ and $15 \%$ w/w) with and without Tween 80 (pH 1.2) 


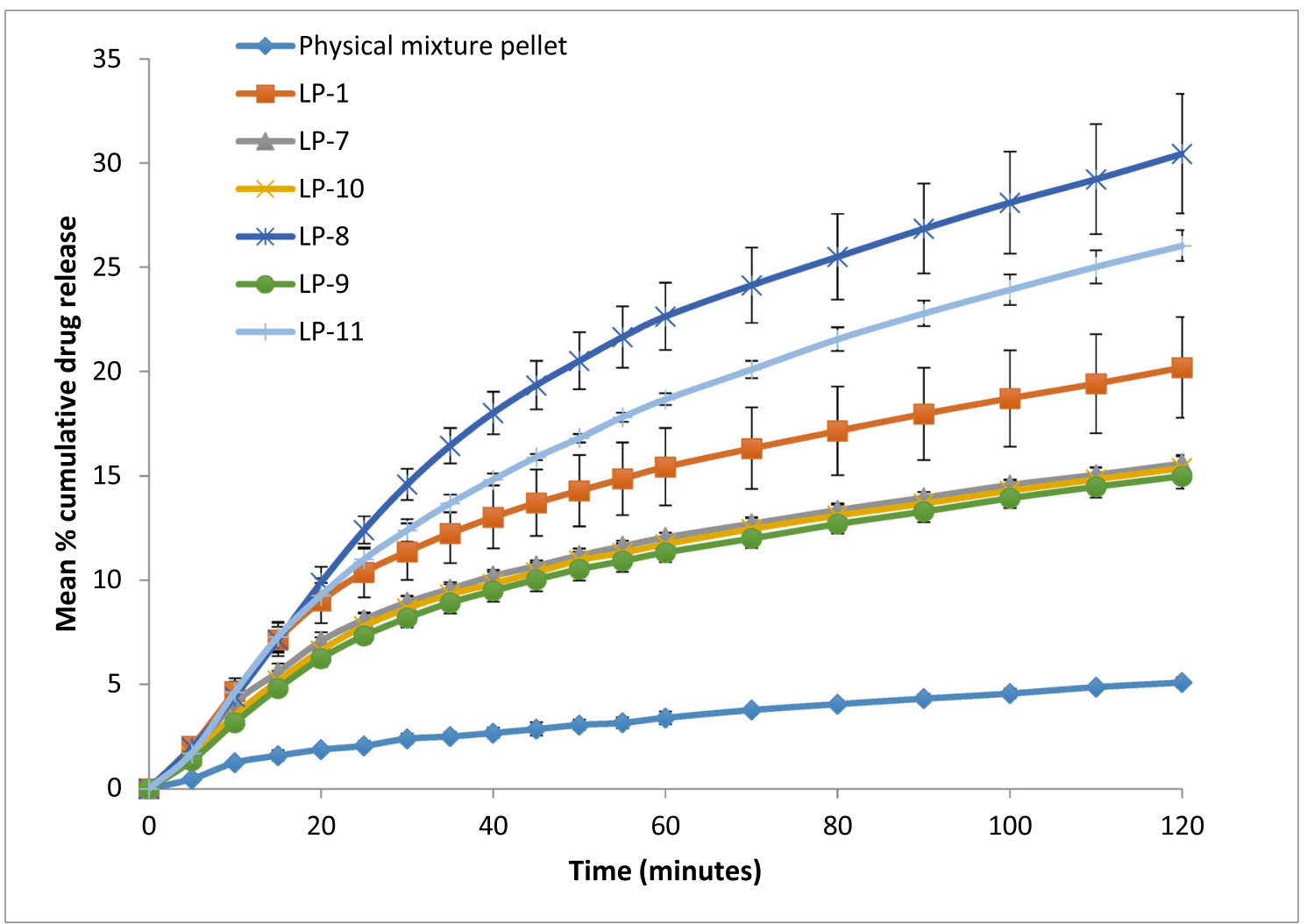

Fig. 5 Dissolution profile of pellets in capsule for naproxen $25 \mathrm{mg}$ with various modifications in attempt to improve dissolution rate (pH 1.2)

at $\times 200$ and $\times 800$ magnification. The formulation LP-8, which contains $38 \%$ Tween 80 , shows a remarkable reduction in surface roughness compared to LP-1. This indicates that surface structure becomes less rough as Tween 80 is increased.

The observation supports the claim made from the previous studies by the authors that co-solvent influences surface structure, which tends to make the surface smoother [1]. It is speculated from the previous studies that liquid vehicle may reduce the crystallinity of the pellet, resulting in smoother surface structure.

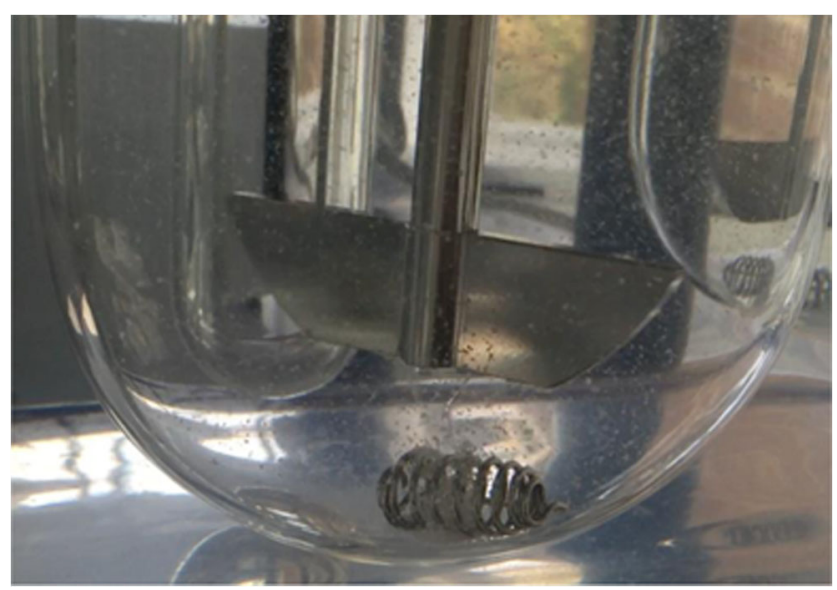

Fig. 6 An image of LP-8 liqui-pellet disintegrate explosively in acidic dissolution medium. Note the small white specks are fragments of the liqui-pellet
The SEM image of formulation LP-11 shows a large number of cracks in the pellet compared to LP-8. In LP-11, which also contains Tween 80 as liquid vehicle, the water content is reduced during the manufacturing of the liqui-pellet. Water and 2-propanol mixture is used instead of just water to reduce overall water content during production. With a reduced amount of water, there will be less bonding within the MCC structure; thus, the pellet quality is reduced. Nonetheless, this is advantageous as the pellet was able to disintegrate well in dissolution medium.

In formulation LP-10, PEG (molecular weight of 1500) and water mixture is used to make the liqui-pellet. It is thought that the PEG at the surface of the pellets will dissolve faster, forming pores. However, it is clear in Fig. 3 image LP- $10^{\text {b }}$, there is no apparent porous structure but the surface did become rougher.

\section{Drug release study}

As mentioned in the previous studies by the authors, naproxen has poor solubility in acidic condition; however, for comparison purpose, the dissolution of liqui-pellets was carried out at $\mathrm{pH}$ 1.2. The authors have found that Tween 80 appears to be the most suitable co-solvent for naproxen liqui-pellet [41]; hence, Tween 80 is the chosen liquid vehicle in this study. As observed in the previous dissolution study of liqui-pellet, 
the lack of disintegrating properties of Avicel led to poor drug dissolution rate [1]. In an attempt to promote disintegration, a superdisintegrant (Primojel) with different concentrations (5, $10,15 \% \mathrm{w} / \mathrm{w}$ ) is introduced into the formulation.

Dissolution test of the formulations containing 5\%,10\% and $15 \% \mathrm{w} / \mathrm{w}$ of Primojel with and without liquid vehicle is shown in Fig. 4. Liqui-pellet formulations containing 5 and $10 \% w / w$ Primojel (LP-1 and LP-2 respectively) had similar dissolution profiles $\left(F_{1}=3.3\right.$ and $\left.F_{2}=97.84\right)$. It can be seen that increasing Primojel concentration to $15 \% \mathrm{w} / \mathrm{w}$ (LP-3) slightly impedes dissolution by $\sim 5 \%$ in comparison to formulations containing 5\% w/w (LP-1) and 10\% $w / w$ (LP-2) Primojel. When comparing LP-1 (Primogel 5\% $w / w$ ) and LP-3 (Primogel 15\% $w / w$ ),$F_{1}=18.91$ and $F_{2}=80.81$. It can be seen the $F_{1}$ value mentioned is above 15 , indicating differences in the drug release profile due; however, the $F_{2}$ value suggests equivalence. If $F_{1}$ is favoured over $F_{2}$ and assuming $15 \%$ Primojel is slowing down drug release compared to $5 \%$ Primojel, this may be due to Primojel forming gel, which can slow down drug release rate. Literature claims that the required concentration of Primojel to achieve optimum disintegration action is $\sim 4 \% w / w$ [43]. Given this claim, Primojel with concentration of $5 \% \mathrm{w} / \mathrm{w}$ is chosen as opposed to $10 \%$ for the other formulations. Results from Fig. 4 confirm formulation without a liquid vehicle has considerably slower drug release rate compared to the one with liquid vehicle $(p<0.05)$. In fact, even with different concentration of Primojel incorporated into the formulation (LP-4, LP-5 and LP-6), the dissolution profiles are similar to that of physical mixture pellet $(p>0.05)$. This further confirms that the characteristic of enhanced drug release in liquisolid formulations can be maintained in liqui-pellet.

After the most suitable liquid vehicle and concentration of Primojel are chosen, various modifications are applied to the formulation to further improve the drug release rate. As seen in Fig. 5, a formulation containing an increased Tween 80 and decreased carrier and coating materials (LP-8) shows the best enhanced drug release profile.

When comparing LP-8 to the non-optimised naproxen liqui-pellet containing Tween 80 (LP-1), it can be seen that the drug release from LP-8 is $\sim 10 \%$ higher $(p<0.05)$ and $\sim 25 \%$ higher than physical mixture pellet $(p<0.05)$ after $2 \mathrm{~h}$. This shows the potential of increasing drug dissolution rate of liqui-pellet when the formulation is optimised. In addition to LP-8 having the fastest drug release rate, its liquid load factor is higher than other formulations $\left(L_{\mathrm{f}}=1.52\right)$, whilst still maintaining excellent-good flow property. With the increase in Tween 80 , less water is required to achieve the appropriate level of plasticity of the extrudate for making quality pellets when spheronised. This is due to the Tween 80 plasticising effect [39]. With less water included in the formulation, it can be deduced that the amount of bonding within the microcrystalline cellulose structure is reduced. Thus, disintegration is more rapid, which is visibly observable during dissolution test (Fig. 6). In fact, the disintegration is rather fast and explosive, which is the reason for higher drug release rate. One of the limitations of microcrystalline cellulose carrier in pelletisation via extrusion-spheronisation is the difficulty of achieving enhanced drug release due to strong bonding, rendering the pellet none disintegrating [44]. In spite of this, microcrystalline cellulose is used because it is the gold standard in extrusion and spheronisation technology as it has the proper rheological properties, cohesiveness and plasticity to yield strong spherical pellets [45]. Formulation LP-11 has the second best enhanced drug release rate $(\sim 26 \%$ within $2 \mathrm{~h})$. This can be explained in a similar manner to LP-8, where 2-propanol and water mixture is used during the liqui-pellet preparation, which effectively reduces the amount of water. Hence, bonding force within microcrystalline cellulose is reduced, leading to improvement in the propensity for disintegration.

It is found that the stage at which Primojel is added during formulation has a slight effect on how well the superdisintegrant performs. When Primojel is added into the liqui-mass system after coating material (LP-7), the drug release rate is $\sim 5 \%$ lower than the same formulation where Primojel is added in the early stage along with the carrier (LP-1). The $F_{1}=27.81$ and $F_{2}=74.83 . F_{1}$ indicates a difference in their dissolution profile but $F_{2}$ indicates equivalence. It should be noted that the range of $F_{1}$ and $F_{2}$ indicating difference or equivalence is only a suggestion from FDA [32] and the basis of the criteria for determining the difference and similarity between dissolution profiles are unclear. In fact, $F_{2}$ is insensitive to the shape of the dissolution profile and is a sample statistic that cannot be used in formulating a statistical hypothesis for the assessment of dissolution similarity [32, 34]. This makes it impossible to evaluate false positive or false negative. With the assumption that $F_{1}$ is reliable, the stages of when Primojel is added does have an influence on drug release rate. It can be seen that the superdisintegrant is added extragranularly in LP-7 and intragranularly in LP-1. Intragranular incorporation of Primojel appears to be more effective than extragranular for improving drug release. This reflects the importance of having an optimum procedure for preparing liqui-pellet. In literature, a combination of intragranular and extragranular incorporation of superdisintegrant is most effective in promoting disintegration [46-48].

In formulation LP-9, Primojel superdisintegrant is replaced by Primellose to see if the sodium starch glycolate or croscarmellose sodium (respectively) will perform better. Results from Fig. 6 shows Primojel (LP-1) have $\sim 4 \%$ more drug release than Primellose (LP-9) after $2 \mathrm{~h}\left(F_{1}=36.94\right.$ and $F_{2}=71.10$ ). If $F_{1}$ is taken into account, then this suggests Primojel is the better superdisintegrant of choice for naproxen liqui-pellet. 


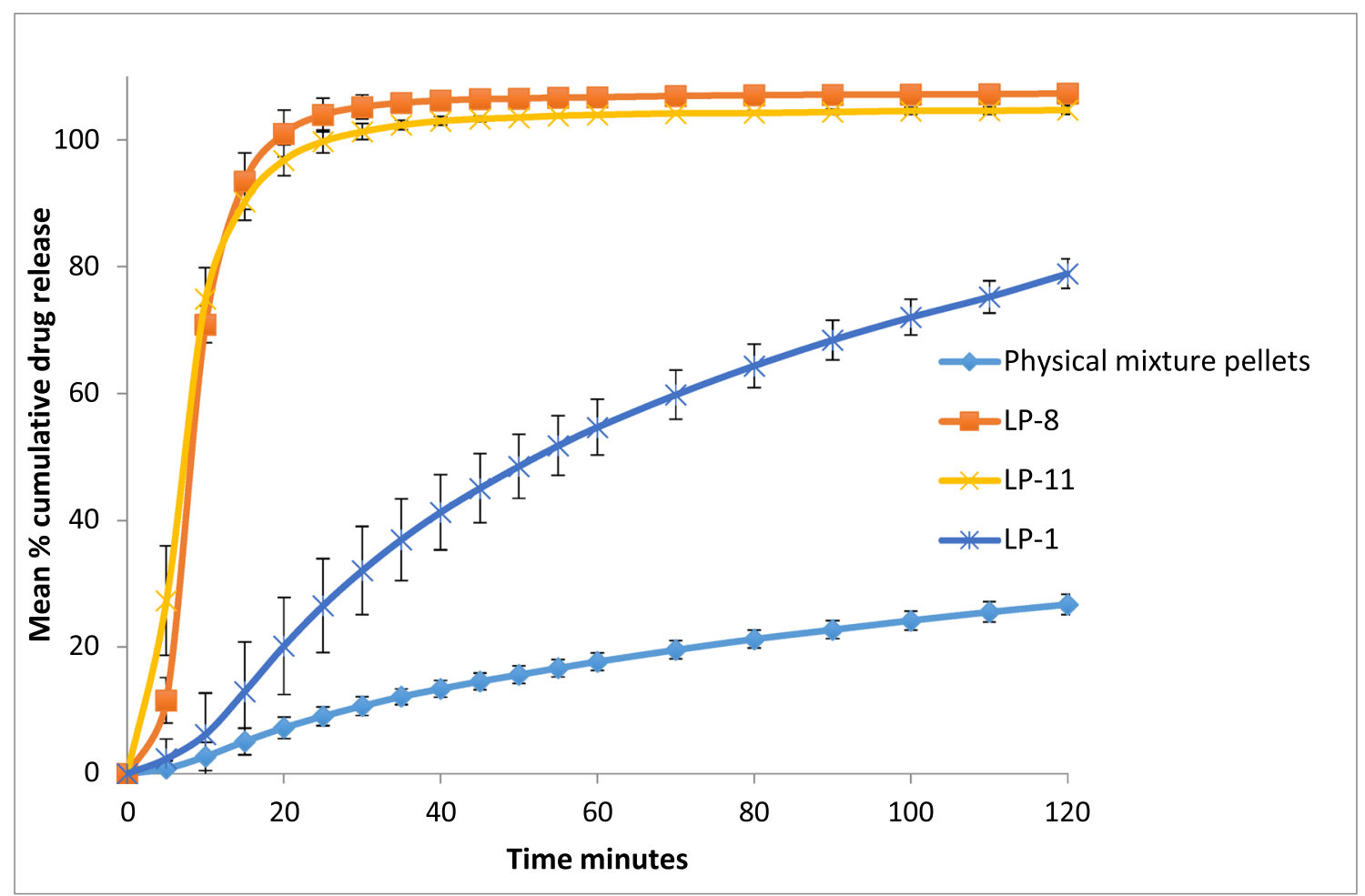

Fig. 7 Dissolution profile of formulations containing naproxen $25 \mathrm{mg}$ with the fastest dissolution rate after modifications, formulation containing Tween 80 as liquid vehicle with Primojel $5 \% \mathrm{w} / \mathrm{w}$, and physical mixture pellets $(\mathrm{pH} 7.4)$

In formulation LP-10, PEG (molecular weight of 1500) and water mixture was used to make the liqui-pellet. It is thought that the PEG at the surface of the pellets will dissolve faster, forming pores which can facilitate the penetration of water into pellets; or that the liquid medication can move out easily via the pores generated as a result of the dissolution of PEG in dissolution medium. However, the results show similar drug release rate to that of LP-7; thus, no improvement in dissolution rate is observed. The SEM results (Fig. 3) show that LP-10 surface is rougher after the dissolution test, but the porous structure is not apparent. Without the porous structure, the drug release rate would not improve.

USP pharmacopoeia suggests performing the dissolution test at $\mathrm{pH} 7.4$ to maintain sink conditions. On the basis of this, the authors believe that those formulations which show higher dissolution at $\mathrm{pH} 1.2$ should exhibit

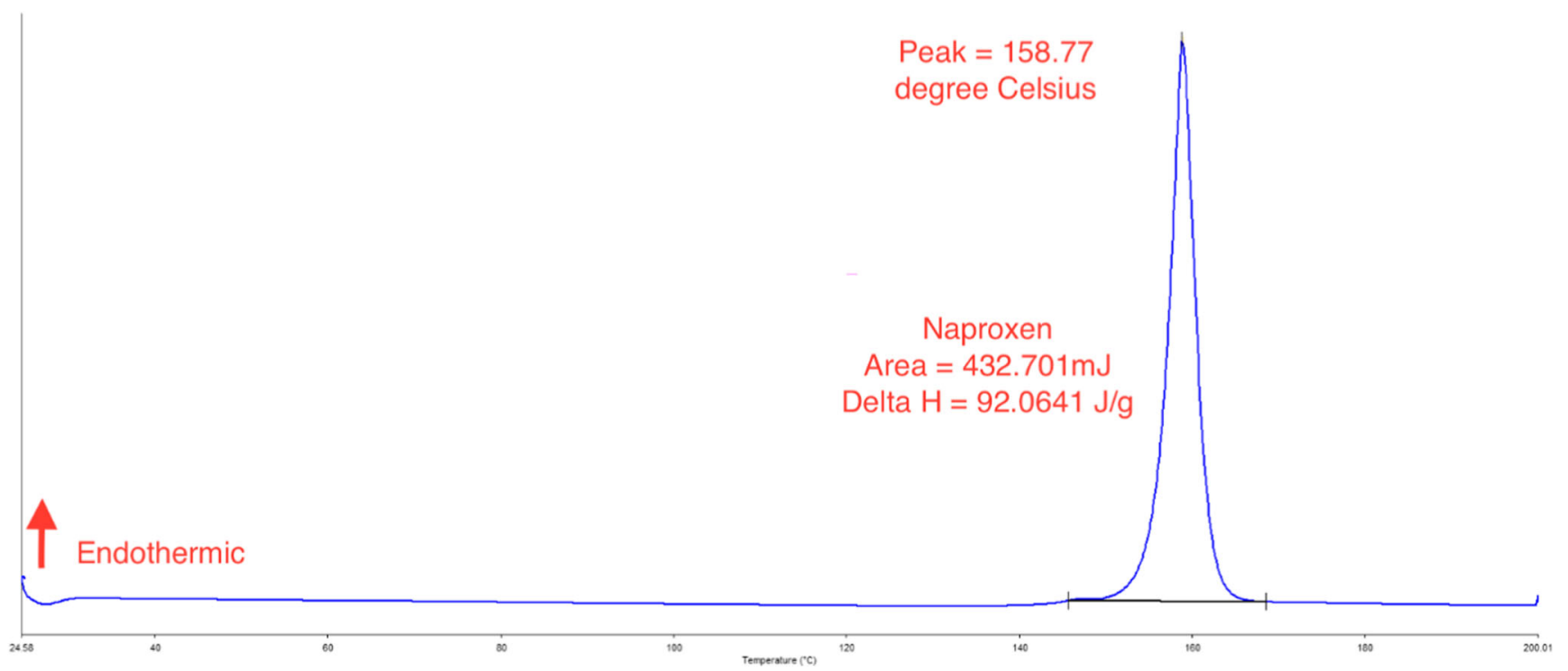

Fig. 8 DSC thermogram of naproxen 


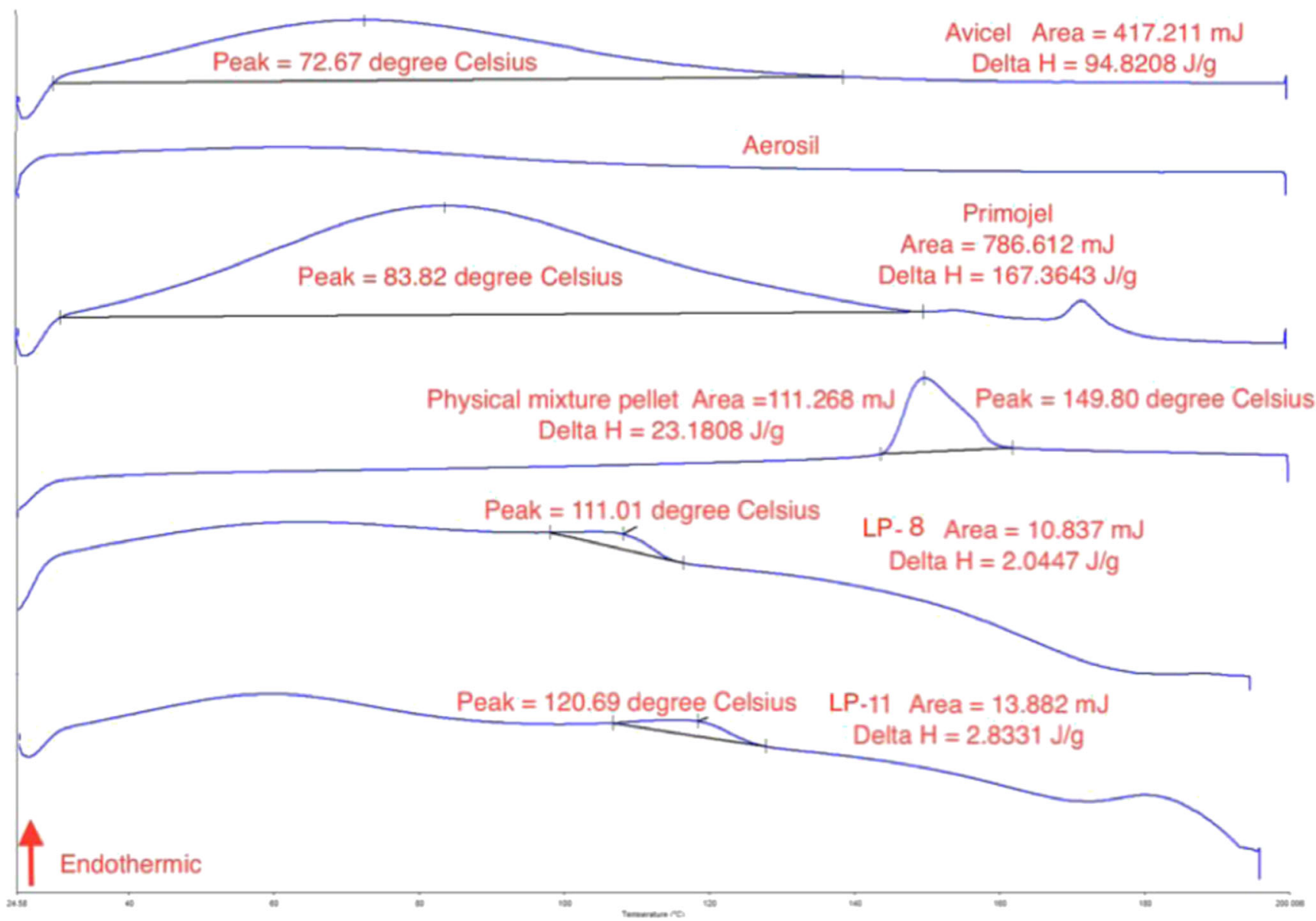

Fig. 9 DSC thermograms of Avicel, Aerosil, Primojel physical mixture pellet, LP-8 and LP-11. Note the scales of Avicel, Aerosil and Primojel are the same but different from physical mixture, LP-8 and LP-11

the better dissolution at higher $\mathrm{pH}$; therefore, only the optimised formulation (LP-8 and LP-11) is selected for dissolution test at $\mathrm{pH} 7.4$ (Fig. 7). The results show both formulations are reaching near plateau after $20 \mathrm{~min}$ ( $\sim 100 \%$ drug release within $20 \mathrm{~min}$ ). This fast dissolution profile is to be expected, as naproxen is a weakly acidic drug; hence, it will dissolve more rapidly in a basic environment. In brief, the results show naproxen liqui-pellet is capable of achieving a fast release rate even though there have been claims that microcrystalline cellulose-based pellets prepared via extrusion-spheronisation tend to prolong drug release [49]. In addition, since the pellets are small, i.e. $\sim 98.8 \%$ of both LP-8 and LP-11 fall into the size of $1 \mathrm{~mm}$ or below; these pellets will undergo gastric emptying relatively fast, similar to liquid [40]. It will be exposed to basic environment relatively quick; thus, drug dissolution should occur faster and potentially improve the drug bioavailability. Also, since the pellets are small, it will be well distributed along the gastrointestinal tract, which could further improve bioavailability [40].

According to results shown in Fig. 6, it seems that water content during the preparation of the liqui-pellet plays a major role in disintegration and drug release rate. It is clear that reducing the water content causes a significant improvement in drug release, most likely due to reduced bonding force within the microcrystalline cellulose structure, which improves disintegration. Furthermore, Avicel has disintegrant properties [43], which are displayed with reduced water content formulations. The drug release rate of the reduced water formulations (LP-8 and LP-11) nearly reached towards the plateau after $20 \mathrm{~min}$, whereas a formulation without reduced water content (LP-1) only has $\sim 79 \%$ drug release after $2 \mathrm{~h}$.

When comparing the results from Figs. 5 and 7 to Tiong et al. studies [36] on naproxen liquisolid compact, liquisolid tablets showed faster drug release rate at $\mathrm{pH}$ 1.2; however, at $\mathrm{pH} 7.4$, the dissolution rate for the optimised formulations are similar or slightly better than Tiong et al. With excellent-good flow property being achieved in liqui-pellet and the intrinsic advantages of liqui-pellet, including possible room for further modifications, the novel liqui-pellet seems like a promising approach in tackling bioavailability issue of poorly watersoluble drugs in a commercially feasible and costeffective way. 


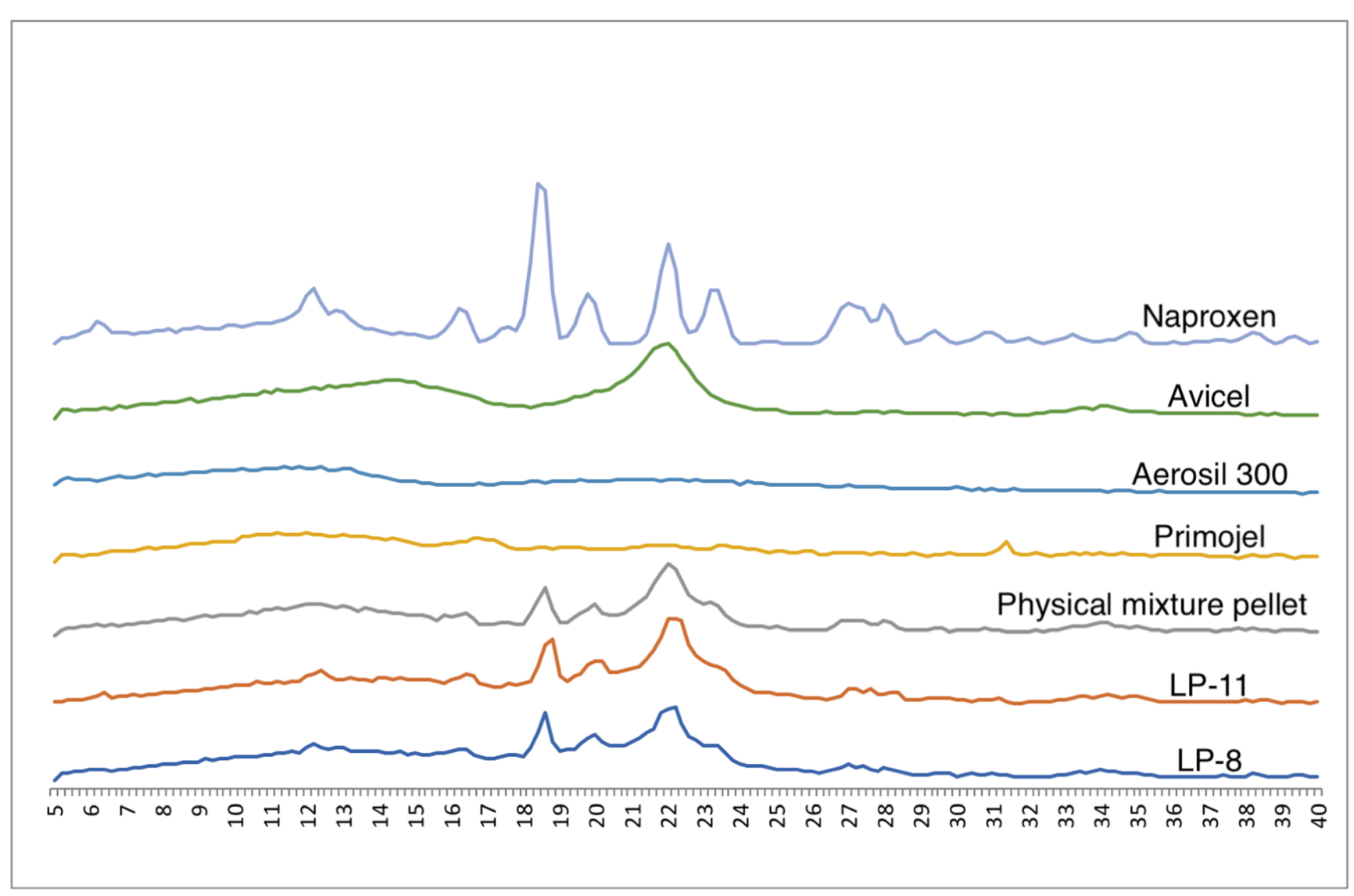

Fig. 10 Diffractograms of naproxen, Avicel, Aerosil, Primojel, physical mixture pellet, LP-8 and LP-11

\section{DSC studies}

The DSC traces of naproxen, Avicel, Aerosil, Primojel, physical mixture pellets and some optimised liqui-pellet formulations are shown in Figs. 8 and 9. The naproxen trace shows a sharp endothermic peak $\left(T_{\mathrm{m}}=158.77^{\circ} \mathrm{C}\right.$ and $\left.\Delta H=92.06 \mathrm{~J} / \mathrm{g}\right)$ indicating its crystalline state. Avicel $\left(T_{\mathrm{m}}=72.67{ }^{\circ} \mathrm{C}\right.$ and $\Delta H=94.82 \mathrm{~J} / \mathrm{g})$ and Primojel $\left(T_{\mathrm{m}}=83.82{ }^{\circ} \mathrm{C}\right.$ and $\Delta H=$ $167.36 \mathrm{~J} / \mathrm{g}$ ) thermograms displayed broad peak. These peaks could be due to water within Avicel and Primojel evaporating, as they are hygroscopic materials. Tiong et al. also observed the evaporation of water from Avicel [36]. As for Aerosil, there was no definitive peak.

When comparing naproxen and physical mixture pellet thermograms as shown in Figs. 8 and 9, it can be seen that there is a small shift of peak from 158.77 to $149.80^{\circ} \mathrm{C}$ respectively. This could be due to Avicel influencing the overall peak of naproxen in the physical mixture pellet. Nonetheless, the crystalline state of naproxen is still present. However, when looking at the DSC traces of optimised formulations (liquipellets), LP-8 $\left(T_{\mathrm{m}}=111.01^{\circ} \mathrm{C}\right.$ and $\left.\Delta H=2.04 \mathrm{~J} / \mathrm{g}\right)$ and LP-11 $\left(T_{\mathrm{m}}=120.69^{\circ} \mathrm{C}\right.$ and $\Delta H=2.83 \mathrm{~J} / \mathrm{g}$ ), the naproxen peak was absent and the $T_{\mathrm{m}}$ lowered, indicating that they were less crystalline and possibly more amorphous, hence the improvement in dissolution.

\section{XRPD studies}

Figure 10 shows major peaks of naproxen at $2 \theta$ values of 12.2 , $16.2,18.4,19.6,22.2,23.2,26.8$ and $27.8^{\circ}$ which are also reported by Maghsoodi et al. [50], with the exception of a sharp peak at $\sim 7^{\circ}$ being present and peak at $26.8^{\circ}$ being absent in Maghsoodi studies. Another research carried out by Mello and Ricci-Junior [51] showed some variation in naproxen peaks between different studies. This could be due to different scan rate settings or the actual state or form of the drug used in various studies. Nonetheless, the general peaks of naproxen are present.

The XRPD diffractogram of physical mixture pellet and formulation LP-8 and LP-11 has no peak other than that of naproxen and Avicel, which indicates no interaction between the excipients and the drug (Fig. 10). As in LP formulations, naproxen is in a molecularly dispersed state; therefore, it is expected to have more halo XRPD compared to the physical mixtures. Figure 9 does not show a big difference in XRPD between the physical mixtures and LP formulations. This could be due to the presence of a high concentration of amorphous Avicel which overshadow the overall XRPD peaks in the physical mixtures. The percentage crystallinity was calculated on the basis of Eqs. 3 and 4 showed that LP8 had the lowest crystallinity (18\% and 36\% using Eqs. 3 and 4 
respectively) compared to physical mixtures which are $23 \%$ and $40 \%$. The authors believed that the crystallinity of the physical mixtures should be much higher than the reported values in this study which could be due to the presence of a high concentration of Avicel in the sample as described earlier.

\section{Conclusion}

It is confirmed that optimised liqui-pellet is capable of enhanced drug release when propensity for disintegration is improved. Although Avicel is known to be non-disintegrating, when the water content is reduced during liqui-pellet production, the pellet is capable of fast and even explosive disintegration. The major drawback of classical liquisolid formulation having poor flowability has been overcome by replacing it with the new liqui-pellet dosage form. All liqui-pellet formulation maintained excellent-good flow properties even with an extremely high liquid load factor of 1.52 , where $38 \%$ of total pellet mass is co-solvent. In conclusion, it is reasonable to postulate that liqui-pellet is highly commercially feasible without having the advantages of liquisolid formulation compromised. Furthermore, there is potential for further optimisation of this novel delivery system as the parameters have yet to be optimised.

Acknowledgements The authors would like to thank Dr. Mohammed Maniruzzaman and Mr. Daniel Holt for their technical assistance.

\section{Compliance with ethical standards}

Conflict of interest The authors declare that they have no conflict of interest.

Open Access This article is distributed under the terms of the Creative Commons Attribution 4.0 International License (http:// creativecommons.org/licenses/by/4.0/), which permits unrestricted use, distribution, and reproduction in any medium, provided you give appropriate credit to the original author(s) and the source, provide a link to the Creative Commons license, and indicate if changes were made.

\section{References}

1. Lam M, Ghafourian T, Nokhodchi A. Liqui-pellet: the emerging next generation oral dosage form which stems from liquisolid concept in combination with pelletization technology. AAPS PharmSciTech. 2019; (in press).

2. Lipinski CA. Drug-like properties and the causes of poor solubility and poor permeability. J Pharmacol Toxicol Methods. 2000;44: 235-49.

3. Spireas S, Sadu S. Enhancement of prednisolone dissolution properties using liquisolid compacts. Int J Pharm. 1998;166:177-88.

4. Loftsson T, Brewster ME. Pharmaceutical applications of cyclodextrins: basic science and product development. J Pharm Pharmacol. 2010;62(11):1607-21.
5. Spireas S, Sadu S, Grover R. In vitro evaluation of hydrocortisone liquisolid tablets. J Pharm Sci. 1998;87:867-72.

6. Nokhodchi A, Hentzschel CM, Leopold CS. Drug release from liquisolid systems: speed it up, slow it down. Expert Opin Drug Deliv. 2011;8:191-205.

7. Hancock BC. Disordered drug delivery: destiny, dynamics and the Deborah number. J Pharm Pharmacol. 2002;54:737-46.

8. Borba PAA, Pinotti M, de Campos CEM, Pezzini BR, Stulzer HK. Sodium alginate as a potential carrier in solid dispersion formulations to enhance dissolution rate and apparent water solubility of \{BCS $\{$ II $\}$ drugs. Carbohydr Polym. 2015;137.

9. Rogers TL, Overhoff KA, Shah P, Santiago P, Yacaman MJ, Johnston KP, et al. Micronized powders of a poorly water soluble drug produced by a spray-freezing into liquid-emulsion process. Eur J Pharm Biopharm. 2003;55:161-72.

10. Rasenack N, Hartenhauer H, Müller BW. Microcrystals for dissolution rate enhancement of poorly water-soluble drugs. Int J Pharm. 2003;254:137-45.

11. Ticehurst MD, Basford PA, Dallman CI, Lukas TM, Marshall PV, Nichols G, et al. Characterisation of the influence of micronisation on the crystallinity and physical stability of revatropate hydrobromide. Int J Pharm. 2000;193:247-59.

12. Kayrak D, Akman U, Hortaçsu Ö. Micronization of Ibuprofen by RESS. J Supercrit Fluids. 2003;26:17-31.

13. Alaei S, Ghasemian E, Vatanara A. Spray drying of cefixime nanosuspension to form stabilized and fast dissolving powder. Powder Technol. 2015;288:241-8.

14. Xu Y, Liu X, Lian R, Zheng S, Yin Z, Lu Y, et al. Enhanced dissolution and oral bioavailability of aripiprazole nanosuspensions prepared by nanoprecipitation/homogenization based on acid-base neutralization. Int J Pharm. 2012;438:287-95.

15. Sugimoto M, Okagaki T, Narisawa S, Koida Y, Nakajima K. Improvement of dissolution characteristics and bioavailability of poorly water-soluble drugs by novel cogrinding method using water-soluble polymer. Int J Pharm. 1998;160:11-9.

16. Barzegar-Jalali M, Valizadeh H, Shadbad MRS, Adibkia K, Mohammadi G, Farahani A, et al. Cogrinding as an approach to enhance dissolution rate of a poorly water-soluble drug (gliclazide). Powder Technol. 2010;197:150-8.

17. Guo B, Liu H, Li Y, Zhao J, Yang D, Wang X, et al. Application of phospholipid complex technique to improve the dissolution and pharmacokinetic of probucol by solvent-evaporation and cogrinding methods. Int J Pharm. 2014;474:50-6.

18. Neslihan Gursoy R, Benita S. Self-emulsifying drug delivery systems (SEDDS) for improved oral delivery of lipophilic drugs. Biomed Pharmacother. 2004;58:173-82.

19. Pouton CW. Formulation of self-emulsifying drug delivery systems. Adv Drug Deliv Rev. 1997;25:47-58.

20. Gullapalli RP. Soft gelatin capsules (softgels). J Pharm Sci. Elsevier Masson SAS; 2010. 99 4107-48 p.

21. Reynolds AD. A new technique for production of spherical particles. Manuf Chem. 1970;41:40.

22. Bechgaard H, Nielsen GH. Controlled-release multiple-units and single-unit doses a literature review. Drug Dev Ind Pharm. 1978;4:53-67.

23. Hu X, Lin C, Chen D, Zhang J, Liu Z, Wu W, et al. Sirolimus solid self-microemulsifying pellets: formulation development, characterization and bioavailability evaluation. Int J Pharm. 2012;438:12333.

24. Kaialy W, Martin GP, Ticehurst MD, Royall P, Mohammad MA, Murphy J, et al. Characterisation and deposition studies of recrystallised lactose from binary mixtures of ethanol / butanol for improved drug delivery from dry powder inhalers. 2011;13:30-43.

25. Mora CP, Martínez F. Solubility of naproxen in several organic solvents at different temperatures. Fluid Phase Equilib. 2007;255: 70-7. 
26. Diffraction X. Standard test method for determination of relative crystallinity of zeolite ZSM-5 by. 2012;i(Reapproved 2011):23-6.

27. Moore JW, Flanner HH. Mathematical comparison of dissolution profiles. Pharm Technol. 1996;20:64-74.

28. O'Hara T, Dunne A, Butler J, Devane J. A review of methods used to compare dissolution profile data. Pharm Sci Technol Today. 1998;1:214-23.

29. FDA. Guidance for industry dissolution testing of immediate. Evaluation [Internet]. 1997;4(August):15-22. Available from: http://www.fda.gov/downloads/Drugs/.../Guidances/ucm070246. pdf. Accessed 10/01/2019.

30. FDA. Guidance for industry guidance for industry, chemistry, manufacturing, and controls; in vitro dissolution testing and in vivo bioequivalence documentation. US Department of Health and Human Services, Food and Drug Administration, Center for Drug Evaluatio. In Vitro. 1997;(September).

31. Adams E, De Maesschalck R, De Spiegeleer B, Vander Heyden Y, Smeyers-Verbeke J, Massart DL. Evaluation of dissolution profiles using principal component analysis. Int J Pharm. 2001;212:41-53.

32. Costa P. An alternative method to the evaluation of similarity factor in dissolution testing. Int J Pharm. 2001;220:77-83.

33. Yuksel N, Kanik AE, Baykara T. Comparison of in vitro dissolution profiles by ANOVA-based, model-dependent and -independent methods. Int J Pharm. 2000;209:57-67.

34. Costa P, Sousa Lobo JM. Modeling and comparison of dissolution profiles. Eur J Pharm Sci. 2001;13:123-33.

35. Pezzini BR, Beringhs AO, Ferraz HG, SegattoSilva MA, Stulzer HK, Sonaglio D. Liquisolid technology applied to pellets: evaluation of the feasibility and dissolution performance using felodipine as a model drug. Chem Eng Res Des. 2016;110:62-9.

36. Tiong N, Elkordy AA. Effects of liquisolid formulations on dissolution of naproxen. Eur J Pharm Biopharm. 2009;73:373-84.

37. Javadzadeh Y, Jafari-Navimipour B, Nokhodchi A. Liquisolid technique for dissolution rate enhancement of a high dose waterinsoluble drug (carbamazepine). Int J Pharm. 2007;341:26-34.

38. Hentzschel CM, Sakmann A, Leopold CS. Suitability of various excipients as carrier and coating materials for liquisolid compacts. Drug Dev Ind Pharm. 2011;37:1200-7.

39. Girdthep S, Punyodom W, Molloy R, Channuan W. Effect of tween 80 on the mechanical and thermal properties of solution-cast blends of poly ( lactic acid ) and cellulose acetate butyrate films. Int Conf Chem Chem Process. 2011;10:95-100.

40. Clarke G, Newton J, Short M. Comparative gastrointestinal transit of pellet systems of varying density. Int J Pharm. 1995;114:1-11.

41. Lam M, Nokhodchi A. European patent no. 1812022.0. Brighton, UK. 2018

42. Aulton ME, Taylor KMG. Aulton's pharmaceutics: the design and manufacture of medicines. Edinburgh: Elsevier Health Sciences; 2013.

43. Rowe RC, Sheskey PJ, Quinn ME, Association AP, Press P. Handbook of pharmaceutical excipients, vol. 6. London: Pharmaceutical Press; 2009.

44. Dukić-Ott A, Remon JP, Foreman P, Vervaet C. Immediate release of poorly soluble drugs from starch-based pellets prepared via extrusion/spheronisation. Eur J Pharm Biopharm. 2007;67:715-24.

45. Dukić-Ott a, Thommes M, Remon JP, Kleinebudde P, Vervaet C. Production of pellets via extrusion-spheronisation without the incorporation of microcrystalline cellulose: a critical review. Eur J Pharm Biopharm. 2009;71:38-46.

46. Mangal M, Thakral S, Goswami M, Ghai P. Superdisintegrants : an updated review. Int J Pharm Pharm Sci Res. 2012;2:26-35.

47. Vandana S, Priyanka S. A review article on: superdisintegrants. J Glob Pharma Technol. 2012;4:15-20.

48. Shihora H, Panda S. Superdisintegrants, utility in dosage forms : a quick review 2011;1:148-53.

49. O'connor RE, Schwartz JB. Spheronization II: drug release from drug-diluent mixtures. Drug Dev Ind Pharm. 1985;11:1837-57.

50. Maghsoodi M. Physicomechanical properties of naproxen-loaded microparticles prepared from Eudragit 1100. AAPS PharmSciTech. 2009;10:120-8.

51. Mello VAD, Ricci-Júnior E. Encapsulation of naproxen in nanostructured system: structural characterization and in vitro release studies. Quim Nova. 2011;34:933-9.

Publisher's note Springer Nature remains neutral with regard to jurisdictional claims in published maps and institutional affiliations. 